\title{
Absence of saturation for finite injected currents in axially symmetric cavity diode
}

\author{
Debabrata Biswas, Raghwendra Kumar and R. R. Puri \\ Theoretical Physics Division \\ Bhabha Atomic Research Centre \\ Mumbai 400 085, INDIA
}

(February 14, 2018)

\begin{abstract}
The Child-Langmuir law is investigated numerically using a fully electromagnetic particle-in-cell code for a closed axially symmetric diode. It is found that the average current transmitted to the anode $\left(J_{\mathrm{TR}}\right)$ increases with the injected current $\left(J_{\mathrm{IN}}\right)$ even after the formation of virtual cathode in both the non-relativistic and relativistic cases. The increase is found to be a power law, $J_{\mathrm{TR}} \sim J_{\mathrm{IN}}^{1-\beta}$. In other words, the time averaged fraction $f$ of electrons reaching the anode varies with the input current as, $f \sim J_{\text {IN }}^{-\beta}$ where $\beta<1$. In contrast, for an infinite parallel plate diode, $f \sim J_{\text {IN }}^{-1}$. The possibility of asymptotic saturation is also discussed.
\end{abstract}

\section{INTRODUCTION}

The Child-Langmuir law is the cornerstone of electron flow in diodes. It gives the maximum current density, $J_{\mathrm{CL}}(1)$, that can be transported from an infinite planar cold cathode (velocity of emission is zero) at zero potential to an infinite planar anode parallel to the cathode at a distance $D$ and potential $V$. In the non-relativistic case, this is given by $[1,2]$

$$
J_{\mathrm{CL}}(1)=\frac{V^{3 / 2}}{D^{2}} \frac{1}{9 \pi}\left(\frac{2 e}{m}\right)^{1 / 2} .
$$

Here, $e$ is the magnitude of the charge and $m_{0}$ the rest mass of an electron.

The Child-Langmuir law is a result of the space-charge effect. As electrons are emitted from the cathode, they gain velocity from the imposed field. At the same time, they experience a repulsive force due to the presence of other electrons. However, all the injected current is transmitted to the anode as long as the injected current, $J_{\text {IN }}$, is less than a particular value, called the critical current $J_{\mathrm{CR}}$. When $J_{\mathrm{IN}}$ exceeds $J_{\mathrm{CR}}$, the net force is such that some of the electrons, instead of moving towards the anode, start moving backwards as if reflected from a "virtual cathode". As a result, only a part of the current, $J_{\mathrm{TR}}$, is transmitted. The position of the virtual cathode depends on $J_{\text {IN }}$ and on the initial velocity, $v_{0}$, of the emitted electrons. In one dimension, $J_{\mathrm{CR}}=J_{\mathrm{CL}}(1)$ in case of cold emission i.e. in case $v_{0}=0$. Also, the virtual cathode is formed on the cathode as soon as the injected current exceeds $J_{\mathrm{CR}}$ and remains there even when $J_{\mathrm{IN}}$ is increased. The transmitted current $J_{\mathrm{TR}}$ in this case is the Child-Langmuir current $J_{\mathrm{CL}}(1)$ for any value of the injected current above $J_{\mathrm{CR}}$. The transition in the transported current is therefore sharp: $J_{\mathrm{TR}}=J_{\mathrm{IN}}$ if $J_{\mathrm{IN}} \leq J_{\mathrm{CR}}, J_{\mathrm{TR}}=J_{\mathrm{CR}}$ if $J_{\mathrm{IN}} \geq J_{\mathrm{CR}}$.

The flow of current below the critical value is steady i.e. time-independent, but is oscillatory above it. The value of the critical current $J_{\mathrm{CR}}$ is thus that value above which the system does not have a steady state. This criterion may be used to evaluate exact analytical expression for $J_{\mathrm{CR}}$ in one-dimensional planar geometry in case the kinetic energy of the electrons is non-relativistic. The analytical expression for $J_{\mathrm{CR}}$ may also be derived in the ultra-relativistic limit.

Since, as mentioned above, the system does not approach any steady state if $J_{\mathrm{IN}}>J_{\mathrm{CR}}$, its behaviour for the input currents exceeding the critical current is described by time-dependent equations. However, it is not possible to solve the time-dependent equations analytically exactly even in one-dimensional planar geometry [3]. Solving time-dependent equations is avoided in the so called classical theory which, for $J_{\mathrm{IN}}>J_{\mathrm{CR}}$, assumes a steady state which takes account of reflections from the virtual cathode. The one-dimensional planar model is analytically exactly solvable within the framework of the classical theory. In particular, it leads to the ChildLangmuir law. Comparison with numerical work shows that the predictions of the classical theory are close to the time-averaged values of physical quantities.

Over the past decade, several studies have been carried out to extend the Child-Langmuir law to two and higher dimensions $[4,5]$. The motivation for this is twofold. In the first place, this helps to benchmark particle-in-cell (PIC) simulation codes. More importantly, the existence of a space charge limited saturation current implies that one need not bother about the material dependent cathode characteristics (the Richardson-Dushman law in case of thermionic emission or the Fowler-Nordheim law [7] for field emission) so long as the injected current is more than what gives rise to the limiting value of the transmitted current. This condition considerably simplifies the simulation of devices that use a diode.

Most studies in two-dimensions have centred around cold emission from a finite emission area in an otherwise infinite parallel plate geometry [4-6]. Note that the Child-Langmuir current is defined in these studies as the current at which oscillatory behaviour sets in (the critical current, $\left.J_{\mathrm{CR}}\right)$. Both numerical and analytical results indicate that this critical current in two-dimensions $\left(J_{C L}(2)\right)$ is higher than the Child-Langmuir current in one-dimension $\left(J_{C L}(1)\right)$. Further, there exists a relation between the two [5]: 


$$
\frac{J_{\mathrm{CL}}(2)}{J_{\mathrm{CL}}(1)}=1+a_{0} \frac{D}{W} .
$$

Here $W$ is width of the emission strip, $D$ is the anodecathode separation and $a_{0} \simeq 0.31$. The question of limiting current (the maximum current transported across the gap) has not however been investigated by these authors.

Recent experiments using photoemission from a cathode have yielded some interesting results [8]. The experiments show that it is possible to transport a much higher current than that predicted by the one-dimensional law for a short emission pulse or limited emitter area and (more importantly) the current may be increased even after formation of the virtual cathode. This leads to a distinction between the critical current (the minimum current required for virtual cathode formation) and the limiting current. In the range of laser intensity studied in these experiments, no limiting current (maximum current that can be transported for a given potential difference and gap separation) was established although the authors suggest the existence of an asymptote.

The question of limiting current in two and higher dimensions thus assumes a great significance since its nonexistence in realistic situations can be a major stumbling block in simulations. With this in mind, in this communication we investigate numerically the Child-Langmuir law in an axially symmetric cavity. We demonstrate that, for axially symmetric closed geometries with a finite emitting area, (i) the current increases even after formation of the virtual cathode, and (ii) the time-averaged fraction of electrons reaching the anode varies as $f \sim J_{\text {IN }}^{-\beta}$ with $\beta<1$ thereby establishing that the transmitted current, $J_{\mathrm{TR}}=f J_{\mathrm{IN}}$, increases as a power law beyond the critical current.

The plan of the paper is as follows: We first review the one-dimensional non-relativistic case and, within the framework of the classical theory, show analytically that above the critical current, the fraction of charges reaching the anode varies inversely with the injected current $(f \sim$ $\left.J_{\text {IN }}^{-1}\right)$. A similar study for the relativistic case is presented next followed by our numerical results for infinite planar diode and axially symmetric cavity diode. Finally our results are summarized in the concluding section. As a matter of convenience, we shall use the notations $J, v$ and $\rho$ to refer to the magnitude of the current density, velocity and charge density respectively.

\section{CRITICAL AND LIMITING CURRENTS: ONE-DIMENSIONAL NON-RELATIVISTIC CASE}

\section{A. Determination of Critical Current}

Consider two infinite plates placed at $z=0$ and $z=D$ at fixed potentials given by

$$
\phi(z=0)=0, \quad \phi(z=D)=V .
$$

Assume that the system evolves to a steady state. In that state, the current at every plane between the plates approaches a value which is independent of time. It can be shown that the potential $\phi(z)$ at the plane at the position $z$ in that case is the solution of the Poisson equation

$$
\frac{\mathrm{d}^{2} \phi}{\mathrm{d} z^{2}}=-4 \pi(-\rho(z))=4 \pi \rho(z)
$$

in which the charge density $\rho(z)$ is determined by the condition that the current density is independent of space so that $\rho(z) v=J_{\text {IN }}$ whereas the velocity $v$ is determined by the energy conservation law $\mathcal{E}_{0}=\frac{1}{2} m_{0} v^{2}-e \phi(z)$ where $\mathcal{E}_{0}=\frac{1}{2} m_{0} v_{0}^{2}-e \phi(0)$ is the initial energy of an electron at the surface of the cathode. Under the condition of cold emission, $v_{0}=0$, and due to the boundary condition (3) at $z=0$, it follows that $\mathcal{E}_{0}=0$. Hence, the law of energy conservation in the steady state reads

$$
\mathcal{E}=\frac{1}{2} m_{0} v^{2}-e \phi(z)=0 .
$$

Equation (4) then assumes the form

$$
\frac{\mathrm{d}^{2} \phi}{\mathrm{d} z^{2}}=\frac{C}{\sqrt{\phi}}
$$

where

$$
C=4 \pi J_{\mathrm{IN}}(m / 2 e)^{1 / 2} .
$$

Equation (6) is to be solved under the boundary conditions given in (3).

The steady state may alternatively be found by solving the equation of motion for electrons [9]. That equation, in the Llewellyn form $[3,10]$, reads

$$
\frac{\mathrm{d}^{3} z\left(t, t_{0}\right)}{\mathrm{d} t^{3}}=-\frac{e}{m_{0}} J_{T}(t)
$$

where $t_{0}$ is the time at which the electron is injected (initial time), $m_{0}$ the rest mass of an electron, $J_{T}=$ $\partial E_{0}(t) / \partial t+4 \pi J$ is the total current and $E_{0}$ is the electric field on the surface of the cathode.

Eq. (8) can be integrated to get

$$
\begin{aligned}
z\left(t, t_{0}\right) & =v_{0}\left(t-t_{0}\right)-\frac{e}{2 m_{0}} E_{0}\left(t_{0}\right)\left(t-t_{0}\right)^{2} \\
& -\frac{e}{2 m_{0}} \int_{t_{0}}^{t} d \tau(t-\tau)^{2} J_{T}(\tau) .
\end{aligned}
$$

In the steady state, $E_{0}(t)=E_{0}\left(t_{0}\right)$ and $\partial E_{0} / \partial t=0$. Also, using the fact that below the critical current, no electron is reflected back, we have $J=J_{\mathrm{IN}}$. Thus,

$$
\begin{aligned}
z\left(t, t_{0}\right) & =v\left(t-t_{0}\right)-\frac{e}{2 m_{0}} E_{0}\left(t_{0}\right)\left(t-t_{0}\right)^{2} \\
& +\frac{4 \pi e J_{\text {IN }}}{6 m_{0}}\left(t-t_{0}\right)^{3}
\end{aligned}
$$

and

$$
v\left(t, t_{0}\right)=v_{0}-\frac{e}{m_{0}} E_{0}\left(t_{0}\right)\left(t-t_{0}\right)+\frac{4 \pi e J_{\mathrm{IN}}}{2 m_{0}}\left(t-t_{0}\right)^{2} .
$$


For the case of cold emission $\left(v_{0}=0\right),(5)$ holds giving $v=\left(2 e \phi / m_{0}\right)^{1 / 2}$ so that the final velocity at the anode is $v_{D}=\left(2 \mathrm{eV} / m_{0}\right)^{1 / 2}$, where $\phi(D)=V$. Let $T$ denote the time that an electron takes to transit the cathode-anode distance $(D)$. Then, with $z=D, v=v_{D}, t-t_{0}=T$, Eqs. (10) and (11) assume the form

$$
\begin{aligned}
L & =-\frac{e}{2 m_{0}} E_{0} T^{2}+\frac{4 \pi e J_{\mathrm{IN}}}{6 m_{0}} T^{3} \\
v_{D} & =\frac{e}{m_{0}} E_{0} T+\frac{4 \pi e J_{\mathrm{IN}}}{2 m_{0}} T^{2}
\end{aligned}
$$

These equations determine the two unknowns, $E_{0}$ and $T$. On eliminating $E_{0}$, the equation for $\mathrm{T}$ in terms of the scaled transit time $\bar{T}$ is

$$
\bar{T}^{3}-\alpha \bar{T}+\alpha=0
$$

where $\alpha=27 V^{3 / 2} / 4 \beta, \bar{T}=T / T_{0}, T_{0}=D /\left(2 m_{0} / e V\right)^{1 / 2}$ and $\beta=9 \pi J_{\mathrm{IN}} D^{2}\left(m_{0} / 2 e\right)^{1 / 2}$. Note that $T_{0}$ is the transit time in the absence of space charge. The condition that all the roots of (14) be real may be shown to be $\alpha>27 / 4$. For $\alpha<27 / 4$, two of the roots are complex while one root is real. The real root is however negative and hence inadmissible as a transit time. Thus $\alpha=27 / 4$ marks the critical current. On substituting the values of $\alpha$ and $\beta$, the expression for the critical current is found to be given by

$$
J_{\mathrm{CR}}=\frac{V^{3 / 2}}{D^{2}} \frac{1}{9 \pi}\left(\frac{2 e}{m_{0}}\right)^{1 / 2} .
$$

Also, note that at $J_{\mathrm{IN}}=J_{\mathrm{CR}}, \bar{T}=3 / 2$ and hence $E_{0}=$ 0 . Thus, at the critical current, the electric field at the cathode vanishes.

\section{B. Determination of Limiting Current : Classical Theory}

The above analysis holds good for $J_{\mathrm{IN}} \leq J_{\mathrm{CR}}$ where the injected and transmitted currents are equal. Beyond the critical current, electrons are reflected and the analysis breaks down. In order to find out what happens beyond the critical current, it is useful to consider yet another steady state model which assumes that a fraction $f$ of the injected electrons is transmitted while the fraction $1-f$ is reflected at some point $z=z_{m}$ so that the transmitted current is $J_{\mathrm{TR}}=f J_{\mathrm{IN}}[2,11]$. Though we have assumed so far that the total energy $\mathcal{E}_{0}$ of an electron entering the diode is zero, it is instructive to carry the classical theory for injected currents greater than the critical currents for the case of non-zero $\mathcal{E}_{0}$. To that end, on invoking the discussion following Eq.(4), the Poisson equation in the two regions, $0 \leq z \leq z_{m}$ and $z_{m} \leq z \leq D$, then assumes the form

$$
\begin{aligned}
& \frac{\mathrm{d}^{2} \bar{\phi}}{\mathrm{d}^{2}}=\frac{4 \alpha(2-f)}{9 \sqrt{\phi}}, \quad 0 \leq z \leq z_{m}, \\
& \frac{\mathrm{d}^{2} \bar{\phi}}{\mathrm{d} \bar{z}^{2}}=\frac{4 \alpha f}{9 \sqrt{\phi}}, \quad z_{m} \leq z \leq D,
\end{aligned}
$$

where $\bar{\phi}=\left(\phi+\mathcal{E}_{0} / e\right) / V, \bar{z}=z / D$, and

$$
\alpha=\frac{9 \pi J_{\mathrm{IN}} D^{2}}{V^{3 / 2}\left(2 e / m_{0}\right)^{1 / 2}} .
$$

Both these equations are of the form $\mathrm{d}^{2} \bar{\phi} / \bar{d} \bar{z}^{2}=A / \bar{\phi}^{1 / 2}$ and can be cast in the form

$$
\frac{\mathrm{d}}{\mathrm{d} \bar{z}}\left(\frac{\mathrm{d} \bar{\phi}}{\mathrm{d} \bar{z}}\right)^{2}=4 A \frac{\mathrm{d} \sqrt{\bar{\phi}}}{\mathrm{d} \bar{z}} .
$$

The solution of this equation reads

$$
\left(\frac{\mathrm{d} \bar{\phi}}{\mathrm{d} \bar{z}}\right)^{2}=4 A \sqrt{\bar{\phi}}+B
$$

Since the electrons come to a stop at $\bar{z}=\bar{z}_{m}$, the law of conservation of energy $\left(\mathcal{E}_{0}=m_{0} v^{2} / 2-e \phi\right)$ implies that $\bar{\phi}\left(\bar{z}_{m}\right)=0$. Also, the field on the electrons at $z=z_{m}$ is assumed to be zero. Hence

$$
\left.\frac{\mathrm{d} \bar{\phi}}{\mathrm{d} \bar{z}}\right|_{\bar{z}=\bar{z}_{m}}=0 .
$$

On using the abovementioned values of $\bar{\phi}$ and $\mathrm{d} \bar{\phi} / \mathrm{d} \bar{z}$ at $\bar{z}_{m}$, it follows from Eq. (20) that $B=0$. Thus

$$
\frac{\mathrm{d} \bar{\phi}}{\mathrm{d} \bar{z}}= \pm 2 \sqrt{A} \bar{\phi}^{1 / 4},
$$

where minus sign holds in the region $0 \leq \bar{z} \leq \bar{z}_{m}$ whereas the plus sign is for the region $\bar{z}_{m} \leq \bar{z} \leq 1$. That is because $\bar{\phi}(z)$ decreases from $\bar{z}=0$ to $\bar{z}=\bar{z}_{m}$ so that the electrons are decelerated and increases beyond $z_{m}$ so that a fraction of electrons is transmitted after they come to rest at $\bar{z}=\bar{z}_{m}$. The solution of (22) yields

$$
\bar{\phi}^{3 / 2}=\frac{9}{4} A\left(\bar{z}-\bar{z}_{m}\right)^{2} .
$$

On using the appropriate values of $A$ in the two regions, the solution can be expressed as

$$
\begin{aligned}
& \bar{\phi}^{3 / 2}=\alpha(2-f)\left(\bar{z}-\bar{z}_{m}\right)^{2}, \quad 0 \leq \bar{z} \leq \bar{z}_{m}, \\
& \bar{\phi}^{3 / 2}=\alpha f\left(\bar{z}-\bar{z}_{m}\right)^{2}, \quad \bar{z}_{m} \leq \bar{z} \leq 1 .
\end{aligned}
$$

Now, on applying the boundary conditions (3), the equations in (24) yield

$$
\begin{aligned}
\left(\frac{\mathcal{E}_{0}}{e V}\right)^{3 / 2} & =\alpha(2-f) \bar{z}_{m}^{2}, \\
\left(\frac{V+\mathcal{E}_{0} / e}{V}\right)^{3 / 2} & =\alpha f\left(1-\bar{z}_{m}\right)^{2} .
\end{aligned}
$$

These equations show that if, $\mathcal{E}_{0}=0$ then

$$
z_{m}=0 \text { and } \alpha f=1 .
$$

The position of the virtual cathode is thus on the cathode when the initial energy of an electron is zero but is away from it otherwise. On substituting for $\alpha$ from (18) in (26), the transmitted current is found to be given by 


$$
J_{\mathrm{TR}}=f J_{\mathrm{IN}}=\frac{V^{3 / 2}}{D^{2}} \frac{1}{9 \pi}\left(\frac{2 e}{m_{0}}\right)^{1 / 2} .
$$

Note that this value of the transmitted current is independent of the injected current and that this expression holds as long as the injected current exceeds the critical current. Eq. (27) is thus the limiting current that can flow through the diode. Note also that the limiting current (27) is identical to the critical current $J_{\mathrm{CR}}$ given in (15).

It should be emphasized that the steady state model for $J_{\mathrm{IN}}>J_{\mathrm{CR}}$ is phenomenological. As is revealed by the numerical solution of the time-dependent equations for injected currents above $J_{\mathrm{CR}}$, the behaviour of the system is oscillatory i.e. it does not approach a steady state. However, the steady state values derived above are found to be in close agreement with the time-averaged values of the numerical results.

\section{CRITICAL AND LIMITING CURRENTS: ONE-DIMENSIONAL RELATIVISTIC CASE}

Finding an explicit expression for the critical current in case of relativistic electronic energies is generally a formidable task. However, an analytical expression for the critical current may be derived in the ultrarelativistic limit in case the initial velocity of the electrons is zero if, following the results of the non-relativistic treatment, we identify the critical current for zero initial velocity as the one for which the electric field at the cathode is zero. We will see that, as in the non-relativistic limit, this current is the same as the limiting current predicted by the classical theory for relativistic energies.

\section{A. Critical Current}

The expression for the critical current in the relativistic case may be derived more conveniently by starting from the Poisson equation (4). The charge density $\rho$ in (4) is determined by $\rho v=J_{\mathrm{IN}}$ and $v$ by the relativistic energy conservation law

$$
m_{0} c^{2}(\gamma(z)-1)-e \phi(z)=0,
$$

where $\gamma(z)=\left(1-v^{2}(z) / c^{2}\right)^{-1}$. Eq. (4) may then be rewritten as

$$
\frac{\mathrm{d}^{2} \gamma(z)}{\mathrm{d} z^{2}}=\frac{K \gamma}{\sqrt{\gamma^{2}(z)-1}}
$$

where $K=4 \pi J_{\mathrm{IN}}\left(e / m_{0} c^{3}\right)$. The boundary conditions (3) in this case assume the form

$$
\gamma(z=0)=1, \quad \gamma(z=D)=1+\frac{e V}{m_{0} c^{2}} \equiv \gamma_{D} .
$$

Following the non-relativistic case, we assume that when the initial velocity of the electrons is zero, the critical value of transmitted current is attained when the field at cathode vanishes. Hence, assuming $\mathrm{d} \phi(z) / \mathrm{d} z=0$ at $z=0$ and invoking also the boundary condition (30) at $z=0$, the integration of (29) gives

$$
\left(\frac{\mathrm{d} \gamma(z)}{\mathrm{d} z}\right)^{2}=2 K\left(\gamma^{2}-1\right)^{1 / 2}
$$

On integrating this equation with the boundary condition (30) at $z=0$ it may be shown that the current density (which, as explained above, is to be identified as the critical current density) is given by

$$
J_{\mathrm{CR}}=\frac{m_{0} c^{3}}{8 \pi e z^{2}} I^{2}(\gamma)
$$

where

$$
I(\gamma)=\int_{1}^{\gamma} \gamma^{-1 / 2}\left(1-\gamma^{-2}\right)^{-1 / 4} \mathrm{~d} \gamma
$$

Eq.(32) determines $\gamma$ implicitly as a function of $z$ and $J_{\mathrm{CR}}$ in case the field at the cathode is zero. However, $J_{\mathrm{CR}}$ is still an unknown. It may be determined by applying the yet unused boundary condition at $z=D$ leading to the relation

$$
J_{\mathrm{CR}}=\frac{m_{0} c^{3}}{8 \pi e D^{2}} I^{2}\left(\gamma_{D}\right)
$$

where $\gamma_{D}$ is given by (30) in terms of the applied voltage $V$. Hence, (34) determines the critical current in terms of known quantities.

The function $I(\gamma)$ in (33) may be evaluated as follows:

$$
\begin{aligned}
I(\gamma) & =\int_{1}^{\gamma} \gamma^{-1 / 2}\left(1-\gamma^{-2}\right)^{-1 / 4} \mathrm{~d} \gamma \\
& =\int_{1}^{\gamma} \mathrm{d} \gamma \sum_{m=0}^{\infty} \frac{\Gamma\left(m+\frac{1}{4}\right) \gamma^{-2 m-1 / 2}}{\Gamma(1 / 4) m !} \\
& =\frac{1}{\Gamma(1 / 4)} \sum_{m=0}^{\infty} \int_{1}^{\gamma} \mathrm{d} \gamma \frac{\Gamma\left(m+\frac{1}{4}\right)}{m !} \gamma^{-2 m-\frac{1}{2}} \\
& =-\frac{1}{2} \frac{\Gamma(1 / 4)}{\Gamma(3 / 4)}\left[\gamma^{1 / 2} \tilde{F}\left(\gamma^{-2}\right)-\tilde{F}(1)\right]
\end{aligned}
$$

where $\tilde{F}(x) \equiv F\left(\frac{1}{4},-\frac{1}{4}, \frac{3}{4} ; x\right)$ stands for the Hypergeometric function. Now, let $\gamma=\gamma_{D}$ and let the voltage be such that $e V>>m_{0} c^{2}$ so that $\gamma_{D}>>1$. Hence, $\tilde{F}\left(\gamma_{D}^{-2}\right) \approx 1$. Using

$$
F(a, b, c ; 1)=\frac{\Gamma(c-a-b) \Gamma(c)}{\Gamma(c-a) \Gamma(c-b)},
$$

and $\Gamma(3 / 4)=1.2254167024$ [12], the expression for the critical current in the ultrarelativistic limit turns out to be given by

$$
J_{\mathrm{CR}}=\frac{m_{0} c^{3}}{2 \pi e D^{2}}\left[\left(1+\frac{e V}{m_{0} c^{2}}\right)^{1 / 2}-0.8471\right]^{2} .
$$

This is in agreement with the result of Jory and Trivelpiece [13] derived under identical boundary conditions though these authors do not identify (37) as the critical current. 


\section{B. Limiting Current}

As in the non-relativistic case, we assume that when $J_{\mathrm{IN}}>J_{\mathrm{CR}}$, the steady state is characterized by a fraction $f$ of particles transmitted beyond the position $z_{m}$ at which the velocity becomes zero so that the current in the region between the cathode and the position $z_{m}$ consists of two parts, the injected current density $J_{\text {IN }}$ moving away from the cathode to the virtual cathode at $z_{m}$ and the reflected part $(1-f) J_{\mathrm{IN}}$ moving to the cathode from the virtual cathode. The Poisson equation in the two regions can then be written as

$$
\begin{aligned}
& \frac{\mathrm{d}^{2} \gamma}{\mathrm{d} z^{2}}=\frac{(2-f) K \gamma}{\sqrt{\gamma^{2}-1}}, \quad 0 \leq z \leq z_{m} \\
& \frac{\mathrm{d}^{2} \gamma}{\mathrm{d} z^{2}}=\frac{f K \gamma}{\sqrt{\gamma^{2}-1}}, \quad z_{m} \leq z \leq D
\end{aligned}
$$

where we have assumed that the energy of the injected electrons is zero. Energy conservation thus leads to the condition $(\gamma-1) m_{0} c^{2}-e \phi=0$. The two equations above are of the same form, whose solution is

$$
\left(\frac{\mathrm{d} \gamma(z)}{\mathrm{d} z}\right)^{2}=2 \tilde{K}\left(\gamma^{2}-1\right)^{1 / 2}+C
$$

where $\tilde{K}=(2-f) K$ for $0 \leq z \leq z_{m}$ and $\tilde{K}=f K$ for $z_{m} \leq z \leq D$. Note that, at $z=z_{m}, \gamma=1, \mathrm{~d} \gamma(z) / \mathrm{d} z=0$. Thus $C=0$. Keeping in mind that the potential decreases in $\left(0, z_{m}\right)$ and increases in $\left(z_{m}, D\right)$, the solutions in the two regions read:

$$
\begin{aligned}
& \int_{1}^{\gamma} \frac{\mathrm{d} \gamma}{\left(\gamma^{2}-1\right)^{1 / 4}}=(2(2-f) K)^{1 / 2}\left(z_{m}-z\right), \quad z \leq z_{m} \\
& \int_{1}^{\gamma} \frac{\mathrm{d} \gamma}{\left(\gamma^{2}-1\right)^{1 / 4}}=(2 f K)^{1 / 2}\left(z-z_{m}\right), \quad z \geq z_{m}
\end{aligned}
$$

Like in the non-relativistic case discussed in the last section, the analysis may be carried for non-zero values of initial energy $\mathcal{E}_{0}$. Restricting, however, to the case of $\mathcal{E}_{0}=0$ with zero initial velocity (so that $\gamma(z=0)=1$ ) and zero potential at the cathode, the boundary conditions give $z_{m}=0$ and

$$
f J_{\mathrm{IN}}=\frac{m_{0} c^{3}}{8 z^{2} \pi e} I^{2}(\gamma)
$$

where $I(\gamma)$ is as in Eq. (33). Eq.(42) with $f J_{\mathrm{IN}} \rightarrow J_{\mathrm{CR}}$ is the same as (32). On applying the boundary condition $\gamma=\gamma_{D}$ at $z=D$ we get the current at the anode. Hence, the transmitted current $f J_{\text {IN }}$ is the same irrespective of the value of the injected current $J_{\text {IN }}$ as long as $J_{\text {IN }}$ is above the critical value at which virtual cathode is formed. The critical value of the current is, therefore, the same as the limiting current. Note from (42) that the fraction of electrons transmitted varies as $f \sim J_{\mathrm{IN}}^{-1}$.

\section{NUMERICAL RESULTS}

The 1-D electrostatic analysis reviewed above indicates that, for cold emission, the critical and limiting currents are identical in both non-relativistic and relativistic cases. The chief motivation of this study is to investigate whether this holds in higher dimension. To this end, we shall use the fully electromagnetic particlein-cell code, SPIFFE [14], and limit ourselves to axially symmetric diodes. For completeness and comparison, we shall first present our numerical results for 1-dimension.

\section{A. One-Dimension: Sheet Model}

In practical terms, the 1-dimensional analysis applies when the cathode and anode are parallel plates of dimensions much larger than the separation between them with uniform emission from the surface of the cathode. A description, convenient for numerical calculations, is to discretize continuous electronic fluid as sheets of uniform charge density parallel to the surface of the two electrodes [3]. The sheets move in the direction perpendicular to their surface. The position $z_{k}$ of the $\mathrm{k}^{\text {th }}$ sheet at time $t$ is governed by the equation [16]

$$
\frac{\mathrm{d} \beta_{k}}{\mathrm{~d} t}=\frac{2}{9} \frac{\alpha_{\mathrm{rel}}}{\gamma_{k}^{3} N}\left[\sum_{i=1}^{M} \bar{z}_{i}-k-\frac{1}{2}\right] .
$$

This equation takes into account the electrostatic repulsion between the sheets. Here $\bar{z}_{k}=z_{k} / D$ is the scaled position of the $k^{\text {th }}$ sheet, $\beta_{k}=v_{k} / c, \alpha_{\text {rel }}=$ $4 \pi J_{\text {IN }} D^{2}\left(9 e / 2 m_{0} c^{3}\right)\left(c / v_{0}\right)^{3}, v_{0}$ is the initial velocity, $N$ is the number of sheets launched per unit transit time in the absence of space charge and $M$ is the total number of sheets present in the diode at any instant of time.

For the numerical calculations presented here, the integration time step $\Delta t$ is $D /\left(200 v_{0}\right), D=0.8 \mathrm{~cm}$ while $N=2000$ for non-relativistic calculations $(V=250 \mathrm{kV})$ and $N=10000$ for relativistic calculations $(V=2 \mathrm{MV})$. These parameters were chosen to satisfy convergence requirements.

As stated before, the system approaches a steady state if the injected current is below its critical value whereas the solution is oscillatory for currents above that value. Since the classical theory is based on the assumption of a steady state even above the critical current, we compare it with the time-averaged behaviour of the physical quantities if the injected current is above the critical current [17].

Fig. 1 is a plot of the transmitted current averaged over $10 \mathrm{~ns}$ as a function of the injected current for $V=250$ $\mathrm{kV}$. It is clear that $J_{\mathrm{TR}}$ attains saturation at $J_{\mathrm{IN}}=$ $4.3 \mathrm{MA} / \mathrm{m}^{2}$. In order to quantify the saturation, we have studied the (time averaged) fraction $f$ of electrons reaching the anode as a function of the injected current. According to the analysis presented in section II, $f \sim J_{\text {IN }}^{-1}$. Fig. 2 confirms that $f \sim J_{\text {IN }}^{-\beta}$ with $\beta=0.999$ 


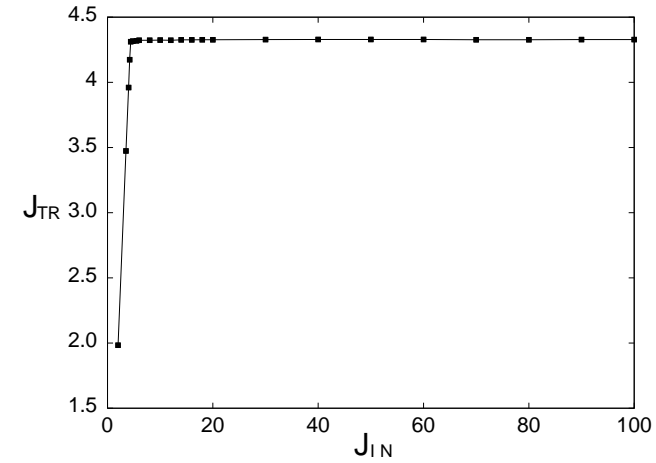

FIG. 1. Time averaged transmitted current density plotted as a function of the injected current density for the 1-dimensional case. Both are measured in $\mathrm{MA} / \mathrm{m}^{2}$. Note the sudden transition at 4.3 where saturation sets in. The theoretical value using Eq. (1) is 4.5.

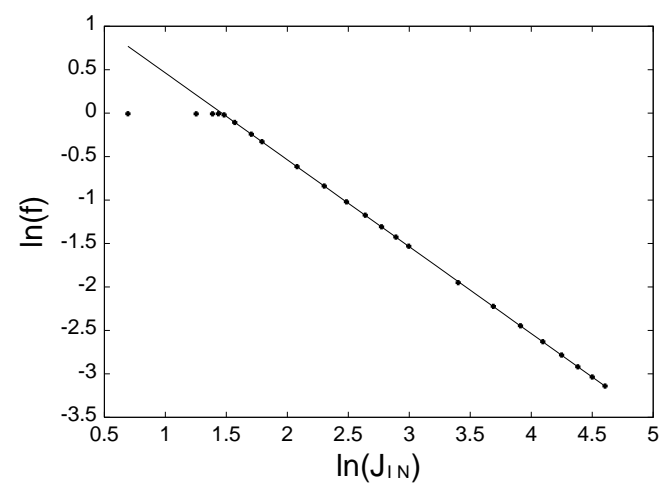

FIG. 2. A log-log plot of the average fraction of transmitted electrons versus the injected current for the 1-dimensional case. The linear fit confirms that $f \sim J_{\text {IN }}^{-\beta}$ with $\beta=0.999$.

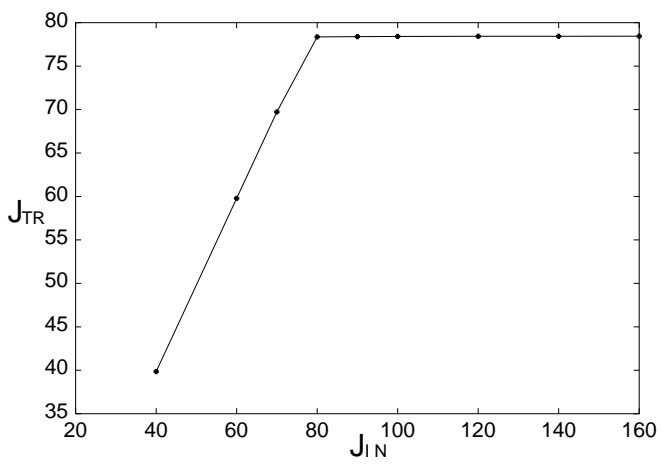

FIG. 3. Time averaged transmitted current density plotted as a function of the injected current density for the 1-dimensional relativistic case. Both are measured in $\mathrm{MA} / \mathrm{m}^{2}$. The critical current density is found to be $78.3 \mathrm{MA} / \mathrm{m}^{2}$ after which saturation sets in. The theoretical value using (37) is $80 \mathrm{MA} / \mathrm{m}^{2}$.

A similar study of the 1-dimensional relativistic case
(2 MV) leads us to an identical conclusion - the critical and limiting currents are indeed identical. Fig. 3 provides confirmation of this. Note that the ultra-relativistic analysis, presented in section III predicts $J_{\mathrm{CR}} \simeq 80 \mathrm{MA} / \mathrm{m}^{2}$ while the observed value is $78.3 \mathrm{MA} / \mathrm{m}^{2}$. The saturation has also been studied using the averaged fraction of transmitted electrons as a function of the injected current. It is found that $f \sim J_{\text {IN }}^{-0.998}$.

Thus, in the 1-dimensional case, the critical and limiting currents are indeed identical and, above the critical current, the fraction of electrons transmitted varies as $f \sim J_{\mathrm{IN}}^{-1}$.

\section{B. Axially Symmetric Diode: PIC}

For the axially symmetric diode, the numerical calculations were performed using the fully electromagnetic particle-in-cell code SPIFFE [14]. The basic algorithm is described below [15].

1. Create the computational mesh and assign metal or vacuum points according to the geometry specified in the input file.

2. Evaluate imposed fields due to potentials applied on metal surfaces

3. Inject macroparticles with specified charge and velocity using the "over-injection" method.

4. Distribute the charge and velocity to the mesh points using a standard weighting scheme.

5. Solve Maxwell's curl equations with the specified boundary conditions (Dirichlet/Neumann) using the Finite Difference Time Domain (FDTD) method.

6. Find forces at the position of the particles by interpolating the field values at the adjacent grid points.

7. Using these forces, find new position and momentum of the particles.

8. Remove particles that reach the end of the simulation region or hit a metal surface.

9. Repeat 1 to 8 .

After a specified number of time steps, it checks if Poisson's equation is satisfied within a specified "error charge". If not, it corrects the electric field after solving Poisson's equation using the error charge.

The geometry of the diode consists of a hollow cylinder of radius $12.5 \mathrm{~cm}$ with the cathode and anode plates of radius $12.5 \mathrm{~cm}$ placed on either end with a separation $D=0.8 \mathrm{~cm}$ between them. The cylinder and anode plate are connected and maintained at a potential, $V$. The cathode plate (on the left in fig. 4) is grounded and the emitting area is restricted to a radius $\mathrm{R}=3.5 \mathrm{~cm}$. In each case, the integration time step was chosen to be 0.0002 ns. Convergence was checked against the mesh spacings in $r$ and $z$ as well as the charge per macro-particle. 


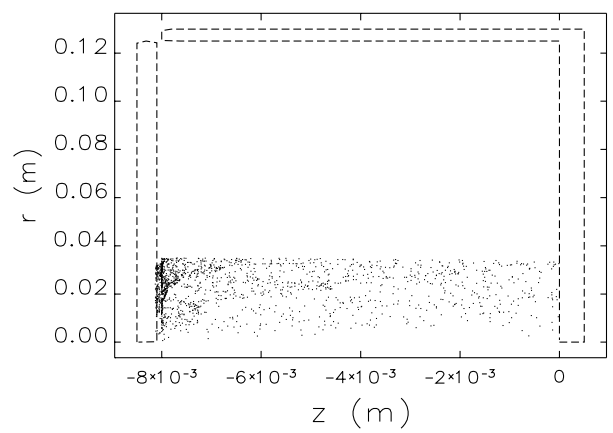

FIG. 4. The geometry of the diode and a typical plot of the particles for $J_{\mathrm{IN}}>J_{\mathrm{CR}}$. Here $J_{\mathrm{IN}}=6 \mathrm{MA} / \mathrm{m}^{2}$ and $V=250$ $\mathrm{kV}$.

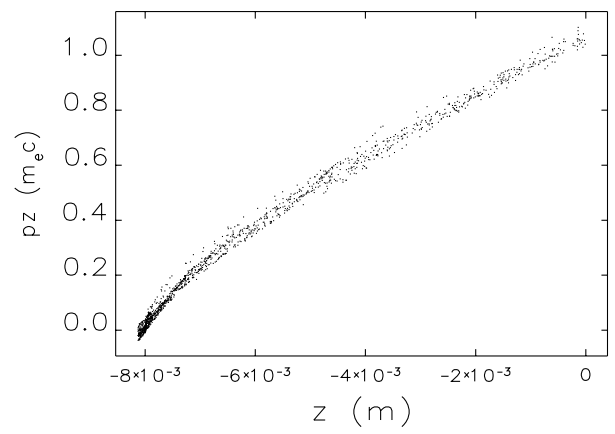

FIG. 5. The phase space plot corresponding to fig. 4. Note that $p_{z}$ is measured in units of $m_{0} c$. In the absence of space charge, $p_{z}=1.1$ at the anode for $V=250 \mathrm{kV}$.

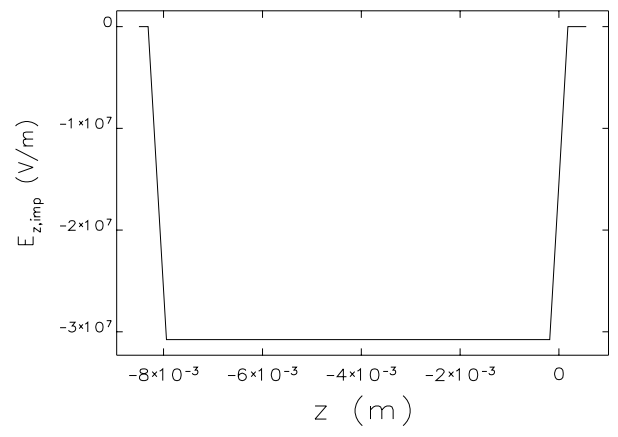

FIG. 6. The imposed electric field, $E_{z, i m p}$ due to the applied potential

The simulation geometry along with a typical plot of the particles in configuration space for $J_{\mathrm{IN}}>J_{\mathrm{CR}}$ is shown in fig. 4 . The phase space plot corresponding to fig. 4 is shown in fig. 5 .

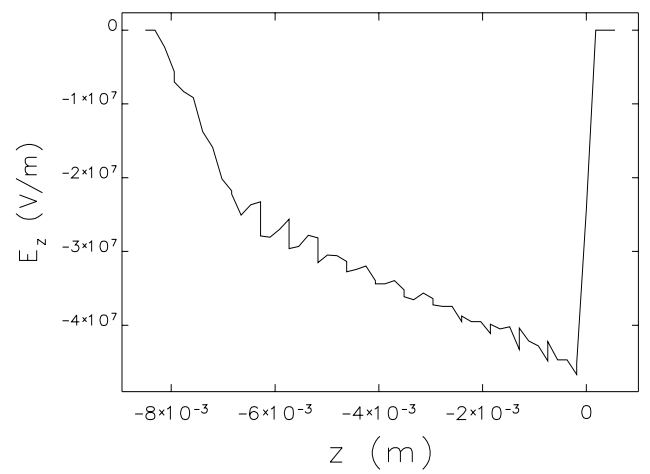

FIG. 7. The electric field after 2 ns. Note that the field at the cathode is nearly zero.

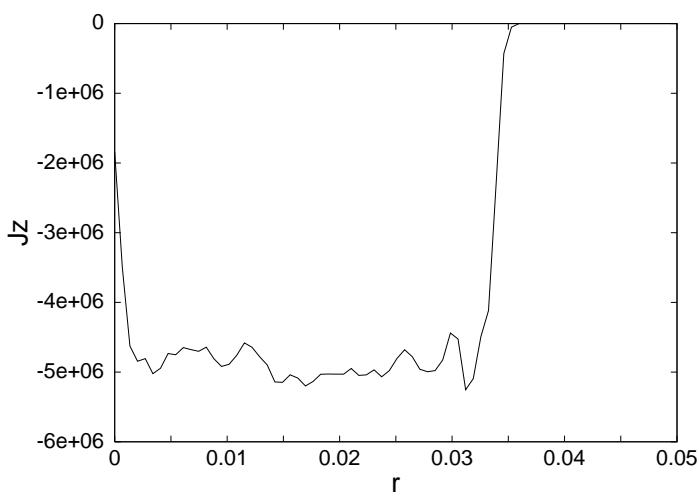

FIG. 8. The time averaged current density close to the anode plotted against the radius. Note that the emitter radius was $0.035 \mathrm{~m}$.

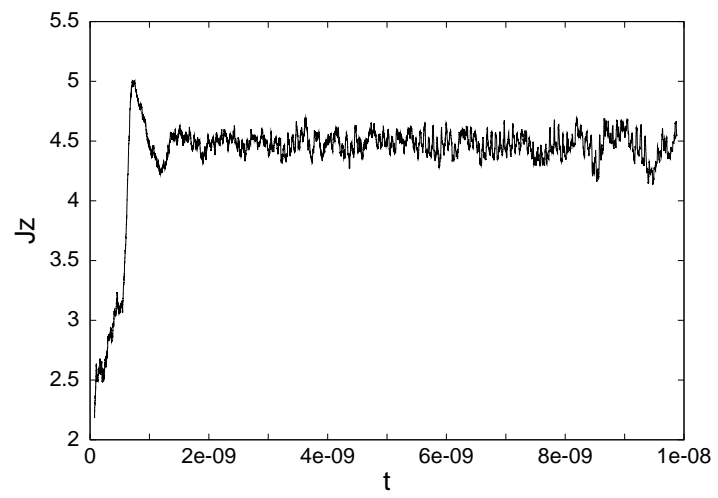

FIG. 9. The locally time averaged current density as a function of time. Note that it is steady after the initial transients.

Figs. 6 and 7 are plots of the electric field $E_{z}$ at the beginning of the simulation and after 2 ns when the transients have settled. Here $J_{\mathrm{IN}}=6 \mathrm{MA} / \mathrm{m}^{2}$ and $r$ is fixed at $0.01 \mathrm{~m}$. Note that in fig. 6 , the field is due to the applied potential alone while in fig. 7 the field is a superposition of the applied field and the electromagnetic field generated by the charges. $E_{z}$ changes little after acquiring the form shown in fig. 7 characterized by its nearly 
vanishing value at the cathode.

The radial profile of the current density for $J_{\mathrm{IN}}=$ $6 \mathrm{MA} / \mathrm{m}^{2}$ is shown in fig. 8 . The plotted value of the $\mathrm{z}$ component of the transmitted current density, $J_{z}$, is averaged over $10 \mathrm{~ns}$ and the section is taken close to the anode. The variation of the radially averaged current density with time is shown in fig. 9. In order to reduce fluctuations, a local time averaging has also been performed.

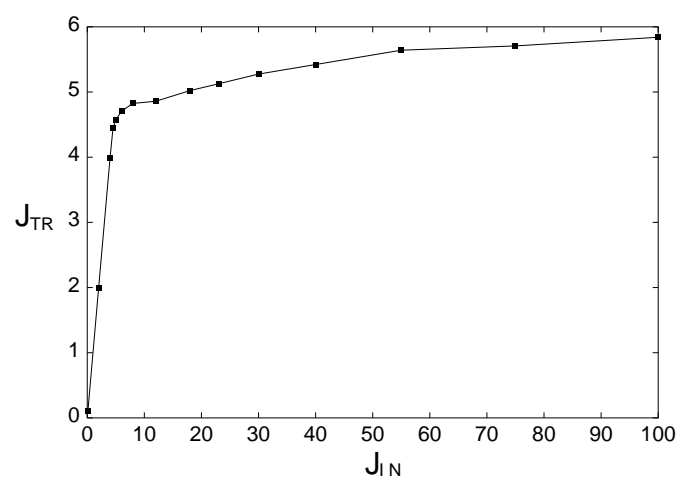

FIG. 10. Time averaged transmitted current density plotted against the injected current density for the axially symmetric diode with $V=250 \mathrm{kV}$. Both are measured in $\mathrm{MA} / \mathrm{m}^{2}$. Unlike the 1-dimensional case, no saturation can be observed for $J_{\mathrm{IN}}>J_{\mathrm{CR}}$.

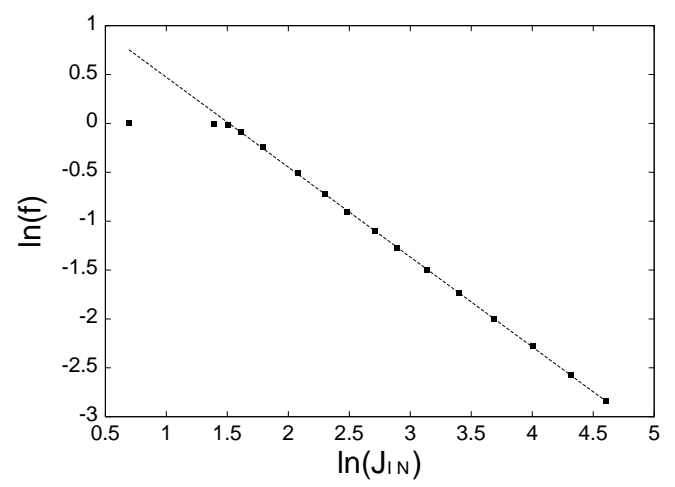

FIG. 11. A log-log plot of the average fraction of transmitted electrons versus the injected current for the axially symmetric diode with $V=250 \mathrm{kV}$. The linear fit for $J_{\text {IN }}>5$ confirms that $f \sim J_{\text {IN }}^{-\beta}$ with $\beta=0.92$.

We now present the central result of this communication. For the non-relativistic studies, the potential considered was $250 \mathrm{kV}$ for which $J_{\mathrm{CL}}(1)=4.57 \mathrm{MA} / \mathrm{m}^{2}$ and $J_{\mathrm{CL}}(2)=1.0354 J_{\mathrm{CL}}(1)=4.73 \mathrm{MA} / \mathrm{m}^{2}$. The injected current was varied in the range $0 \leq J_{\text {IN }}<100$. While criticality sets in at around $J_{\mathrm{CL}}(1)$, there appears to be no saturation in this range (see Fig. 10). While $J_{\mathrm{TR}} \simeq J_{\mathrm{IN}}$ for $J_{\mathrm{IN}}<J_{\mathrm{CR}}$, the transmitted current increases as a power law beyond the critical current. This is evident from a plot of the average fraction of electrons reaching the anode as a function of the injected current (Fig. 11). The power law behaviour is evident with $f \sim J_{\text {IN }}^{-0.92}$. Thus the transmitted current increases beyond the critical current as $J_{\mathrm{TR}}=f J_{\mathrm{IN}} \sim J_{\mathrm{IN}}^{0.08}$.

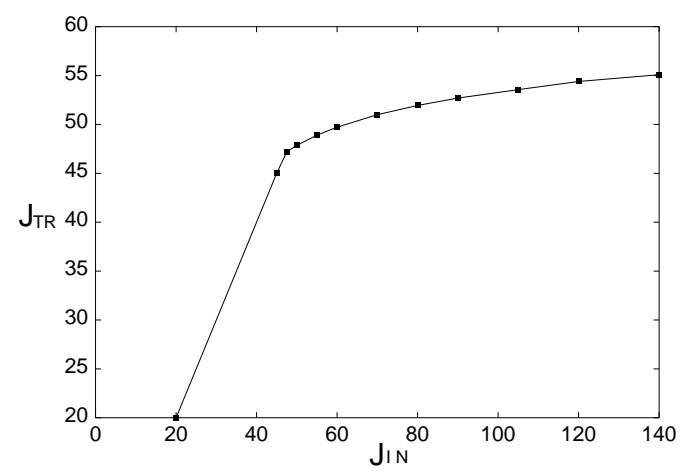

FIG. 12. Time averaged transmitted current density plotted against the injected current density for $V=2 \mathrm{MV}$. Both are measured in $\mathrm{MA} / \mathrm{m}^{2}$.

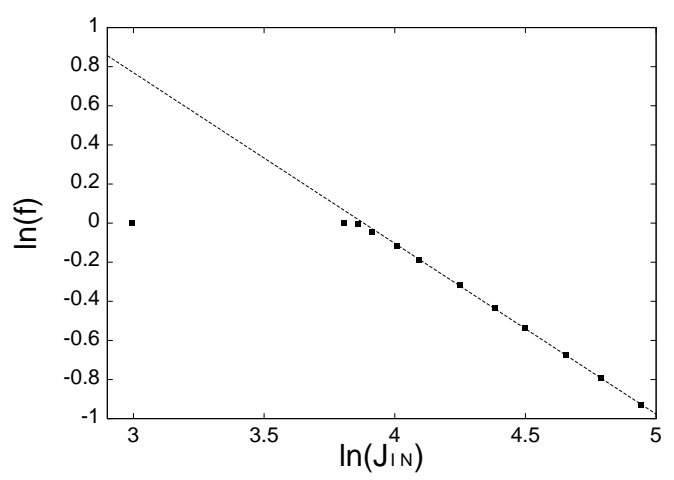

FIG. 13. A log-log plot of the average fraction of transmitted electrons versus the injected current for $\mathrm{V}=2 \mathrm{MV}$. The fit is made for $J_{\mathrm{IN}}>50$ and the value of $\beta$ is 0.873 .

A similar study for a relativistic case (2 MV) leads us to an identical conclusion - the transmitted current increases beyond the critical current as evident from Fig. 12. The power law behaviour can be seen from a plot of $\ln (f)$ vs $\ln \left(J_{\mathrm{IN}}\right)$ (Fig. 13) and for this particular case, $\beta=0.873$. Thus, $J_{\mathrm{TR}} \sim J_{\mathrm{IN}}^{0.127}$. Note that in the relativistic case, there is a strong pinching effect due to the self magnetic field. The observed value of the critical current, $J_{\mathrm{CR}}$, is $47 \mathrm{MA} / \mathrm{m}^{2}$ which is much less than the 1-dimensional prediction $\left(80 \mathrm{MA} / \mathrm{m}^{2}\right)$.

These results do not preclude the existence of asymptotic saturation. Rather, a fit of $J_{\mathrm{TR}}$ using the form $J_{\mathrm{SAT}}-A / J_{\mathrm{IN}}^{\gamma}$ provides a marginally better fit than the power law. However, a unique set of values for $\left(J_{\mathrm{SAT}}, A, \gamma\right)$ is hard to determine with the existing data as several sets exist with nearly the same error (sum of squares). Nevertheless, it is clear from these studies that $J_{\mathrm{TR}}$ does not saturate for finite values of injected currents beyond the critical value. 


\section{CONCLUSIONS}

The existence of a limiting current in an axially symmetric cavity diode has been investigated. It is found that $J_{\mathrm{TR}}$ does not saturate with an increase in $J_{\mathrm{IN}}$ beyond the critical value characterised by the onset of reflection. This is unlike the case of one-dimensional diode which exhibits saturation of $J_{\mathrm{TR}}$ as soon as $J_{\mathrm{IN}}$ exceeds $J_{\mathrm{CR}}$. Our main result is the power law behaviour, established through numerical computations between the transmitted current $J_{\mathrm{TR}}$ and finite values of the injected current $J_{\mathrm{IN}}$ in an axially symmetric diode operating above the critical current $J_{\mathrm{CR}}$. The exponent depends on the voltage. We have also explored the possibility of asymptotic saturation and found that there exist sets of parameters $\left(J_{\mathrm{SAT}}, A, \gamma\right)$ for which the form $J_{\mathrm{TR}}=J_{\mathrm{SAT}}-A / J_{\mathrm{IN}}^{\gamma}$ provides a marginally better fit than the power law $J_{\mathrm{TR}} \sim J_{\mathrm{IN}}^{\beta}$.

The findings here thus have an important bearing on numerical simulations which generally assume saturation of transmitted current even in two-dimensional diodes at a finite value of the injected currenbt. Analytical and numerical studies of a one-dimensional diode have also been presented for the sake of comparison and completeness.

[1] C. D. Child, Phys. Rev. 32, 492 (1911).

[2] I. Langmuir, Phys. Rev. 21, 419 (1923).

[3] C. K. Birdsall and W. B. Bridges, Electron Dynamics of Diode Regions (Academic Press, New York, 1966).

[4] J. W. Luginsland, Y. Y. Lau and R. M. Gilgenbach, Phys. Rev. Lett. 77, 4668 (1996); J. W. Luginsland, Y. Y. Lau, R. J. Umstattd and J. J. Watrous, Phys. Plasmas 9, 2371 (2002). J. J. Watrous, J. W. Luginsland, M. H. Frese, Phys. Plasmas 8, 4202 (2001).

[5] Y. Y. Lau, Phys. Rev. Lett. 87, 278301 (2001);

[6] K. G. Kostov and J. J. Barroso, Phys. Plasmas 9, 1039 (2002).

[7] R. .H. Fowler and L. Nordheim, Proc. R. Soc. London Ser. A 119, 173 (1928).

[8] A. Valfells, D. W. Feldman, M. Virgo, P. G. O'Shea and Y. Y. Lau, Phys. Plasmas 9, 2377 (2002).

[9] see also V. Akimov, H. Schmel, H. Kolinsky, A. Ya. Ender and V. I. Kuznetsov, Phys. Plasmas 8, 3788 (2001).

[10] F. B. LLwellyn, Electron inertia Effect (Cambridge University Press, London, 1941).

[11] A similar analysis for drift space can be found in [3].

[12] M. Abramowitz and A. Stegun, Handbook of Mathematical Functions with Formulas, graphs and Mathematical Tables, (National Bureau of Standards, United States Dept. of Commerce, 1972).

[13] H. R. Jory and A. W. Trivelpiece, J. App. Phys. 40, 3924 (1969).

[14] SPIFFE is written by M. Borland and available along with the user guide at http://www.aps.anl.gov

[15] M. Borland, Summary of equations and methods used in SPIFFE, APS/IN/LINAC/92-2, 29, June 1992.
[16] For the non-relativistic equation of motion, see [3].

[17] In each case, time averaging is performed after eliminating transients. 